\title{
Final Analysis Dataset
}

National Cancer Institute

\section{Source}

National Cancer Institute. Final Analysis Dataset. NCI Thesaurus. Code C115492.

The collection of data used for the final analysis of results for a clinical trial. 